

\section{Cultura entre las manos: Una opción de comunicación para el desarrollo de la comunidad de Sordos de La}

\section{Habana}

Cultura entre las manos: A communication option for Havana's deaf community development

Dor: $10.15213 /$ redes.n10.p255

YALENA GISPERT, GLADYS GONZÁLEZ, HILDA SALADRIGAS

\section{ABSTRACT}

In the present investigation identified from ethnography and taking as fundamental analysis concerning the Communication for Development and Social Mediation Paradigm and the conceptual approaches and the relationship between them and the media, culture, Deaf community heritage and how the Cultura entre las Manos project has mediated the development of the community of Deaf Havana. From the description and characterization of the actions of this communication exercise, lacking practical precedents and academics in the panorama of Special Education, Social Communication and cultural-heritage sector in Cuba, and the study of evolution levels of information and knowledge on issues of tangible and intangible assets in Deaf people, changes in the expression and dialogue capacity, and levels of integration and inclusion and social group achieved after participation in the project, the study shows an evaluative analysis of how "Cultura entre las Manos" media the development of Deaf people.

KEYWORDS: SOCIOCULTURALDEVELOPMENT, MEDIATION, DEAFCOMMUNITY, PARTICIPATION, CULTURA ENTRE LAS MANOS 


\section{RESUMEN}

En la presente investigación se identifica, desde la etnografía y tomando como referentes fundamentales de análisis la Comunicación para el desarrollo y el Paradigma de la Mediación Social, así como las aproximaciones conceptuales y la relación entre estos y los medios de comunicación, la cultura, el patrimonio y la comunidad Sorda,cómo el proyecto Cultura entre las manos ha mediado el desarrollo de la comunidad de Sordos de la capital cubana. A partir de la descripción y tipificación de las acciones de este ejercicio de comunicación, que carece de precedentes prácticos y académicos en el panorama de la Educación Especial, la Comunicación Social y el ámbito cultural-patrimonial en Cuba, así como el estudio de la evolución de los niveles de información y conocimiento sobre cuestiones del patrimonio tangible e intangible en las personas Sordas, los cambios en la capacidad expresiva y de diálogo, y los niveles de integración e inclusión grupal y social alcanzados tras la participación en el Proyecto, el estudio muestra un análisis valorativo de la forma en que Cultura entre las manos media el desarrollo de las personas Sordas.

PALABRAS CLAVES: DESARROLLO SOCIOCULTURAL, MEDIACIONES, COMUNIDAD SORDA, PARTICIPACIÓN, CULTURA ENTRE LAS MANOS

\section{INTRODUCCIÓN}

La atención a las prácticas comunicativas que genera una emisora de radio implica un ejercicio que, por la capacidad de cobertura de este medio de comunicación de masas, abarque un gran espectro de dimensiones de análisis.

Por tales razones se requiere un análisis más integral, en función de lo que las personas pueden hacer con la información pública a que acceden las audiencias radiales y de lo que la institución-emisora radial está en condiciones de crear para potenciar espacios inclusivos para todos, tomando en cuenta a aquellos que coexisten con bajos niveles de información y no son públicos de este medio de comunicación por su discapacidad: la minoría lingüística/cultural de las personas Sordas. Se trata, entonces de realizar un análisis y valoración justa, sin exceso de optimismo pero sin determinismos fatalistas anticipados que contribuyan a enriquecer las prácticas comunicativas que tienen lugar en este sentido y aventurar posibles iniciativas que continúen democratizando el acceso a la comunicación y la información. 
A pesar de la pertinencia que posee la indagación acerca de la capacidad que posee un medio de comunicación como la radio para potenciar el desarrollo social y humano de personas que no forman parte de las tradicionales audiencias de este medio, no existen antecedentes investigativos sobre esta temática en Cuba. En alguna medida, los estudios relacionados, son trabajos que en su mayoría responden a tesis (de licenciatura, maestría y doctorado) que si bien contribuyen al fomento y desarrollo de la Lengua de Señas Cubana y al reconocimiento social de la comunidad Sorda como minoría Lingüística, no involucran de manera explícita la comunicación, ni los medios de comunicación de masas, como agentes de desarrollo sociocultural de esta comunidad. Solo en relación con lo mediático se localizó un estudio realizado por el Centro de Investigaciones Sociales (cIs), sobre la implementación del sistema de Closed Caption en Cuba.

De ahí que el acercamiento a las investigaciones realizadas sobre esta comunidad indica que las que con más frecuencia se han realizado son aquellas de naturaleza pedagógica y, más próximo a nuestros días, las de corte lingüístico.

\subsection{INVESTIGACIONES EXISTENTES DESDE EL CAMPO PEDAGÓGICO}

En la educación de las personas Sordas ha sido una polémica histórica el empleo de las diferentes tendencias educativas, principalmente entre el oralismo y el gestualismo. Hechos que evidencian la incomprensión de la esencia de la Sordera y sus implicaciones socioeducativas.

Como antecedentes históricos de una concepción pedagógica cada vez más humanista, que considera la pluralidad lingüística y cultural, se destacan los expresados por Rosa M. Castellanos y Xiomara Rodríguez (Castellanos y Rodríguez, 2003). En el primero, la autora hace una periodización de cómo se concebía la persona sorda en las diferentes formaciones económico sociales, valorándose desde una perspectiva humanista hasta nuestros días. En el segundo, la autora sistematiza cinco etapas en el desarrollo histórico de la pedagogía para la educación a estas personas, que incluyen desde la mitad del siglo xvi hasta la fecha, las que parten del empleo de diversas metodologías, el gestualismo, el oralismo, métodos que consideran la comunicación total, el bimodalismo y por último el bilingüismo.

Por otra parte, estos antecedentes abordan el comportamiento educativo en el ámbito internacional. Estos referentes son muy importantes para el análisis del desarrollo de la educación de estas personas, pero están centrados en los métodos de comunicación utilizados. 
En cambio, la indagación realizada por la Dra. Luisa América Toledo Martínez, compendia el devenir histórico de la educación de las personas Sordas en Cuba desde 1959 hasta el año 2005. La autora, sistematiza los fundamentos teóricos que sustentan el desarrollo de la educación de las personas Sordas en la Isla, los cuales coinciden, con las concepciones acerca de la sordera y las personas Sordas en el ámbito internacional. Entre ellos describe las tendencias por las que ha transitado la enseñanza de los Sordos en Cuba hasta llegar al uso de la Lengua de Señas como primera lengua, lo cual describe como modelo al que se aspira para facilitar el desarrollo personal y social de los discapacitados auditivos.

Asimismo, destaca los modos de comunicar y educar con la participación de la familia, la Asociación Nacional de Sordos de Cuba (ANsoc), el Ministerio del Trabajo y Seguridad Social, y de otras instituciones junto a la escuela.

Las aristas de esta investigación y otras del ámbito pedagógico realizadas a partir de 1994 - año en que el Ministerio de Educación de Cuba aprueba la utilización de la Legua de Señas en las escuelas como legua de instrucción hasta la fecha, se circunscriben a constatar el uso de la Lengua de Señas Cubana como primera lengua de la comunidad Sorda, y por ende el medio ideal para comunicar, informar e instruir más allá del ámbito educativo. Aspecto que desde la fecha es necesario contemplar en toda acción que pretenda potenciar el desarrollo y la inserción social de los miembros de esta comunidad.

Más tarde, en el año 2007, como parte del proyecto bilingüe cubano de la comunidad Sorda - que tuvo entre sus iniciadoras en Cuba la Dra. Xiomara Rodríguez Fleitas, junto a un reconocido grupo de profesionales -, el Lic. Alejandro Marzo, actual Presidente de la Asociación Nacional de Sordos de Cuba (ANSOC), abordó una temática de gran valor para este grupo social al caracterizar la identidad de la comunidad Sorda como grupo social minoritario con peculiaridades que lo distinguen de otros: lengua propia, costumbres, tradiciones, historia y cultura como comunidad lingüística particular. Investigación que promueve el proceso de integración social a partir de la formación de una identidad bilingüe-bicultural.

\subsection{INVESTIGACIONES DESDE EL CAMPO LINGÜÍSTICO}

Las investigaciones realizadas sobre el análisis lingüístico de la Lengua de Señas Cubana (LSC) desde la década de los 90 hasta el presente, se concentraron básicamente en los siguientes aspectos: 
1. El análisis de repertorios de palabras de la LSc, con la elaboración concreta de un manual de lengua de señas, que tuvo su primera edición en julio de 1993, hasta llegar a la actual Biblioteca Virtual de la Lsc, presentada en 2009 por la Asociación Nacional de Sordos de Cuba (ANSOC).

2. La descripción de las características estructurales de las señas, con el estudio del nivel de las configuraciones manuales.

3. La realización de trabajos con vistas a lograr la construcción de modelos lingüísticos propios de la Lengua de Señas Cubana.

En el análisis de repertorios de palabras de la LSC uno de los primeros trabajos (1994) fue asumido por la Dra. Marlen Domínguez de la Facultad de Artes y Letras de la Universidad de La Habana, quien tituló su estudio Un breve análisis de la Lengua de Señas Cubana. En él realiza un acercamiento a la estructura interna de las señas como signo lingüístico.

Con posterioridad tuvo lugar la descripción de las características estructurales de las señas, con el estudio del nivel de las configuraciones manuales, que se inició con la investigación de la Lic. Clara Valenciaga (2003) y que permitió conocer y comprender el valor de los componentes manuales y no manuales para el proceso de enseñanza y aprendizaje de la Lengua de Señas Cubana. Esta investigación fue sucedida por la descripción de las configuraciones manuales más frecuentes de la LSC (2005).

En el año 2007 la psicóloga Lourdes Padilla en su estudio El significado de los componentes manuales de la lengua de señas cubana como sistema lingüístico refirió los elementos fundamentales del desarrollo y origen de la Lengua de Señas Cubana, así como de las exigencias didácticas para el trabajo con sus significados.

En estos esfuerzos investigativos se incluyen también los aportes de la Lic. Heysel Pérez (2004) quien distingue el signo gestual a partir de los parámetros formativos quinésicos que son aislables y que tienen, en muchos casos, valor diferencial con múltiples variantes, al entrar en una combinación simultánea en el espacio. Los parámetros articulatorios descritos por esta investigadora son tres: configuración manual, lugar de articulación y el movimiento.

En el primer componente registra tres elementos importantes: región de contacto de la mano durante el movimiento, orientación de la configuración manual según la palma de la mano y la disposición, atendiendo al uso de las manos y las reglas básicas de simetría y asimetría, que caracterizan esta lengua en la que muchos autores reconocen el valor de apoyo de una mano y el valor de realización de la seña en la otra, lo cual explica la simetría y la asimetría. 
Las investigaciones realizadas en esta dirección han permitido también el reconocimiento de la dactilología como un componente real de la comunicación entre los Sordos en la actualidad. Más allá del criterio de su surgimiento para fines educativos, se afirma que la dactilología es una parte constituyente de las lenguas de señas de todo el mundo.

Otro componente con frecuencia descrito es el de la expresión corporal y facial, como elementos de la Lengua de Señas, que en la bibliografía más actualizada aparecen como valores del escenario del discurso de dichas lenguas.

Por lo antes descrito, concerniente al campo de la lingüística, podemos inferir que la estructuración de la Legua de Señas Cubana y los modelos lingǘsticos que la soportan, han comenzado a ganar espacio dentro de las investigaciones científicas en Cuba, en aras del reconocimiento social de esta lengua y su desarrollo. Aspectos que aunque no forman parte de los objetivos de esta investigación manifiestamente, son guías en la producción de prácticas comunicativas que afectan a la comunidad Sorda, para de esta forma contribuir al estudio y salvaguarda de su patrimonio lingüístico.

\subsection{APORTES DE LA INVESTIGACIÓN DESDE LO MEDIÁTICO}

La investigación desde lo mediático se observa en el medio televisivo, pasados 3 años de la aplicación del sistema de Closed Caption a un grupo programas de la televisión cubana. Otro soporte con potencialidades para la viabilidad para la comunidad que es la prensa impresa no se ha estudiado y, por su puesto, mucho menos la radio.

En lo concerniente a la participación de la televisión en la política cubana referida a las personas con discapacidad, la Asociación Cubana de Limitados Físico-Motores (ACLIFIM), la Asociación Nacional de Ciegos (ANCI) y la Asociación Nacional de Sordos (ANSOc), firmaron el 4 de agosto de 1998 un acuerdo con el Instituto Cubano de Radio y Televisión (ICRT) que tuvo como principal lineamiento la inclusión del tema de las personas con discapacidad dentro de la Política de Programación de la Radio y la Televisión Cubanas, con el fin de que estos medios contribuyeran a la integración activa de esas personas a la sociedad y coadyuvaran a la transformación de las actitudes sociales del resto de los miembros de la sociedad para con este grupo de personas.

En relación con las personas Sordas específicamente, se realizaron esfuerzos dirigidos a la implementación del sistema de Closed Caption en Cuba, en el año 2002. El Centro de Investigaciones Sociales (cis) 
del Instituto Cubano de Radio y Televisión (ICRT) fue el que realizó una evaluación tras su implementación. La aceptación que hasta junio de 2005 tuvo esta tecnología en la comunidad de Sordos constituyó el centro del estudio.

Marta Lilia Sánchez Ortega y Patricia Yamilet Gutiérrez — ambas investigadoras del cis - estudiaron los resultados de la puesta en marcha del subtitulaje oculto hasta ese momento. Determinaron la periodicidad del uso y los niveles de satisfacción de los usuarios del Closed Caption, así como las sugerencias y necesidades de la comunidad de Sordos.

Como resultado se obtuvo que la mayoría de los Sordos e Hipoacúsicos entrevistados, utilizan el Closed Caption. De ellos, gran parte lo emplea todos los días de la semana y están medianamente satisfechos con el servicio. Desean ver ampliado el servicio, lo consideran muy útil porque les ayuda a incrementar el vocabulario aunque proponen además, la implementación de la Lengua de Señas en los espacios televisivos.

Las propuestas principales de los sujetos para mejorar el Closed Caption se pueden entender como necesidad de elevar los niveles de información y su calidad, para que de manera certera pueda traducirse su aplicación y uso en una importante contribución a la eliminación de las barreras de comunicación en Cuba y por ende la elevación de la cultura, máxima expresión del desarrollo social.

Luego de este recorrido se identificó como problema de investigación: ¿Cómo el proyecto Cultura entre las manos que lleva a cabo Habana Radio, emisora de la Oficina del Historiador de la Ciudad de La Habana, ha mediado el desarrollo de la comunidad de Sordos de la capital?

Los objetivos de la investigación fueron, en relación con el proyecto Cultura entre las manos que lleva a cabo Habana Radio, emisora de la Oficina del Historiador de la Ciudad de La Habana, demostrar su valor como programa de comunicación para el desarrollo, establecer el modo en que ha mediado el desarrollo de la comunidad de Sordos de la capital.

Para el acercamiento al objeto de estudio se partió de la Premisa de que el proyecto Cultura entre las manos que impulsa Habana Radio, emisora de la Oficina del Historiador de la Ciudad de La Habana durante poco más de cinco años, ha empleado la interacción comunicativa grupal e interpersonal para desarrollar una cultura hacia la preservación del patrimonio material e inmaterial.

Ello ha incrementado los niveles de información y conocimiento en materia histórico patrimonial de los miembros de dicha comunidad, ha fortalecido su capacidad expresiva, ha facilitado los intercambios entre los 
individuos que componen la comunidad, y ha robustecido la integración de ellos como tejido social.

Por sus características y resultados, dicho proyecto puede ser considerado como un programa de comunicación para el desarrollo con muchas potencialidades aún por revelar.

Se asumieron como categorías analíticas: Comunicación para el Desarrollo y las Mediaciones. En la definición de Comunicación para el desarrollo tomaremos como referente el concepto ofrecido por el comunicólogo Luís Ramiro Beltrán para quien la

"Comunicación de apoyo al desarrollo es el uso de los medios de comunicación - masivos, interpersonales o mixtos - como factor instrumental para el logro de las metas prácticas de instituciones que ejecutan proyectos específicos en pos del desarrollo económico y social.” (2005, p. 10)

A los efectos de la investigación estos factores serían el uso de medios de comunicación - interpersonales y grupales - , desde una emisora de radio con carácter comunitario, mediante acciones participativas en pos de lograr el desarrollo de una cultura hacia la preservación del patrimonio tangible e intangible y con ello potenciar mejores niveles de integración y socialización en una comunidad lingüísticamente minoritaria.

En este sentido se valora Cultura entre las manos como programa de Comunicación para el Desarrollo Sociocultural a partir dela evaluación de los niveles de información, la ampliación de la capacidad expresiva, la integración grupal y la inclusión.

$\mathrm{Al}$ analizar estas condicionantes podemos introducir en las definiciones conceptuales las Mediaciones,ya que al comprender como fuentes del desarrollo las acciones comunicativas generadas a partir de la interacción con las personas Sordas por razón del fomento de la cultura, entendemos la capacidad mediadora del proyecto Cultura entre las manos.

Tomando como fundamento teórico principal el Paradigma de la Mediación que aporta el estudioso español Manuel Martín Serrano, podemos explicar las relaciones que existen entre el cambio social y la producción simbólica. Asumir esta teoría implica entender que las mediaciones producen modelos de ajuste al cambio social, porque

"desde el punto de vista cognitivo la mediación equivaldría al sistema de reglas y de operaciones aplicadas a cualquier 
conjunto de hechos, o de cosas pertenecientes a planos heterogéneos de la realidad, para introducir un orden." (Serrano, 2008, p. 49)

De igual modo,

"las mediaciones hay que entenderlas como procesos estructurantes que provienen de diversas fuentes, que inciden en los procesos de comunicación y conforman las interacciones comunicativas de los actores sociales." (Orozco, 2002, p. 26)

En tal sentido, entenderemos que Cultura entre las manos puede mediar cognitiva y estructuralmente el desarrollo de una cultura hacia la preservación del patrimonio tangible e intangible y con ello potenciar mejores niveles de integración y socialización en una comunidad lingüísticamente minoritaria desde:

1. La participación de los Sordos en espacios de intercambio grupal creados para la transmisión de valores y conocimiento.

2. La interacción social en el entorno patrimonial-cultural a partir de facilitar los enlaces e intercambios entre los miembros de la comunidad Sorda y la red de Instituciones del Centro Histórico de la Ciudad.

3. La aportación de los miembros de esta comunidad en la búsqueda de soluciones para la accesibilidad.

La investigación, se centra en el estudio de diferentes comunidades - en este caso una minoritaria (la Sorda) y la otra mayoritaria (la oyente) - , por lo que se toma en consideración que se debe tener un conocimiento sensible de las pautas culturales (de la comunidad de la cual no es parte el investigador), además de los conocimientos científicos.

Cuando un investigador, de una comunidad $\mathrm{u}$ otra, se plantea investigar las prácticas comunicativas que generan el desarrollo de una de ellas, se enfrenta a un tipo de investigación intercultural. Y, hay que tener en cuenta entonces las "diferencias" que hacen a su vez los puntos de comunión de los miembros de la comunidad en cuestión.

En el caso de la investigación de la comunidad Sorda realizada por un oyente, serían los diferentes ritmos de tiempo, la connotación del espacio, importancia de la información visual, preferencias, aspiraciones, intereses, modos de organización, formas de acercarse y diferencias de lengua entre ambas culturas. 


\section{DISEÑO DE INVESTIGACIÓN}

Para dar cuenta de esta diversidad y dar solución al problema de investigación se seleccionó, dentro del registro de métodos cualitativos el Método Etnográfico ya que como define Jesús Galindo posee gran perspectiva descriptiva y en gran medida, su resultado está en manos del observador. Dentro del catálogo de métodos de investigación social "depende menos de instrumentos de registro y medición que otras técnicas de investigación” (Galindo, 1998, p. 350).

No se privilegian como fuentes los documentos o textos escritos de antemano, sino que para llegar al conocimiento del otro el investigador intenta llegar primero hasta ese otro, lo cual presupone la inserción en la comunidad de Sordos en este caso, para alcanzar la necesaria objetividad científica de la investigación. Aunque esto no significa que los aportes de otros investigadores no sean tomados en cuenta, solo que es válido resaltar que cuando se les utiliza como únicas fuentes para llegar al más cercano conocimiento de una comunidad proporcionan datos muy limitados (Massone, 2012).

La revisión bibliográfica y documental entonces, fue seleccionada como técnica de la investigación para lograr un acercamiento teórico al tema, así como a la definición de los referentes y contextos en que se desarrolla el proyecto Cultura entre las manos.

Es importante resaltar que la propuesta de la etnografía, para estudiar el desarrollo de las prácticas sociales de la comunidad Sorda, exhorta a los investigadores a ser conscientes de cómo nuestra propia subjetividad puede estar influyendo en el análisis de los datos.

Como explica María I. Massone (2012), la objetividad reside en ser conscientes de nuestra propia subjetividad y para lograr este fin entendemos que es primordial utilizar métodos para el análisis. Como asegura esta autora, en los estudios de la comunidad Sorda realizados por personas, que aunque se identifiquen con su cultura, forman parte de la gran mayoría convergen dos mundos de significación diferentes que el lingüista (investigador) oyente debe considerar (...) a fin de no dar por resultado versiones oyentes de estas lenguas (esta cultura) (Massone, 2012).

Por este motivo se empleó como técnica un sistema de observación participante y no participante, de campo, abierta y no estandarizada con el principal objetivo de aproximarnos a las relaciones que existen entre la comunidad Sorda de La Habana que acude a las acciones del proyecto Cultura entre las manos, entre los propios miembros de la comunidad y su evolución.

De igual manera se acudió a la entrevista a expertos y actores porque como técnica de obtención de información constituye una fuente de significado. El 
objetivo de esta técnica fue acceder a la visión que tienen los expertos y actores sobre las relaciones que se establecen a través de Cultura entre las manos, así como indagar sobre las posibles capacidades desarrolladas. Las entrevistas fueron en profundidad sobre la base de cuestionarios abiertos no estructurados.

La unidad de análisis de la presente investigación fue laComunidad de Sordos que participa en el proyecto, la cual oscila entre 80 y 130 sujetos de los diferentes municipios de La Habana.

Teniendo en cuenta los diferentes enfoques que tiene el trabajo con la comunidad Sorda se pueden identificar un conjunto de actores, relaciones y fuerzas, que han permitido que se vean o no los avances en el desarrollo social y humano de la comunidad Sorda.

Como actores tenemos clara la presencia de la comunidad Sorda organizada en su Asociación Nacional de Sordos. De otra parte el Ministerio de Educación, el cual apoya, gestiona, y lidera varias de las iniciativas para el desarrollo. Y la academia, acompañada por los desarrollos teóricos y entendiendo las necesidades de la comunidad Sorda, aclarando que este abordaje es uno de los tantos que busca responder a las necesidades de este grupo, pero que no es la única respuesta que la sociedad debe brindar a las personas con limitación auditiva. Además, se encuentran los especialistas en Patrimonio y Comunicación que desde su labor han colaborado con la realización de Cultura entre las manos.

En este sentido vale la pena aclarar, si se quiere que las acciones llevadas a cabo hasta el momento tengan verdadera influencia en la inclusión social y la participación ciudadana de las personas con discapacidad - en el caso que nos corresponde: la comunidad Sorda - , se necesitarán nuevos indicadores de análisis. Uno de ellos podría estar, precisamente, en el terreno comunicacional de acceso al capital simbólico de la cultura.

Reconocer la diferencia asienta el compromiso de iniciar el recorrido y existe la confianza de que en virtud del diálogo intercultural constituya una garantía el acceso a la información, la comunicación y la cultura, lo cual en nuestro caso parte de un medio de comunicación como la radio, lo que representa una experiencia inédita en Cuba, un elemento poco estudiado que puede despertar intereses investigativos desde el área de la comunicación.

\section{MÁS dE CINCO AÑOS DE TRABAJO EN POS DEL DESARROLLO SOCIOCULTURAL Y LA INTEGRACIÓN DE LA COMUNIDAD SORDA DE LA HABANA}

Cuando se escucha por primera vez la formulación de la idea de un proyecto 
que va al encuentro de las necesidades e intereses culturales de las personas Sordas y que, a su vez, tiene entre sus gestores principales a una emisora de radio, se siente el temor de apresurarse en emitir un juicio sobre una situación aparentemente paradójica que sólo el acercamiento y conocimiento de los objetivos y el desarrollo del proyecto pueden superar, ya que justamente la plataforma sonora propia del diseño de una emisora radial podría ser la causa para que las personas Sordas continuasen al margen de los mensajes e informaciones que este poderoso medio de comunicación trasmite.

Precisamente fuera del público habitual de la emisora Habana Radio se encuentra la comunidad de Sordos. Realidad que sirvió de estímulo a un grupo de profesionales, sensibilizados con la cotidiana desinformación de la comunidad Sorda, para pretender lograr un acercamiento, brindándoles a sus miembros una nueva opción de conocimiento e incentivo por la identidad nacional.

Así nació, hace más de 5 años, el proyecto Cultura entre las manos: un programa sociocultural de educación patrimonial para el fomento del diálogo intercultural entre la comunidad minoritaria Sorda y la comunidad mayoritaria de oyentes que comparten la ciudad. Asimismo, este proyecto suma a su vocación por promover y difundir el patrimonio habanero y nacional, la convicción de la necesidad de preservar, proteger y fomentar el conocimiento del patrimonio propio de ese grupo humano: la Lengua de Señas Cubana.

Esta práctica comunicacional apuesta por promover la gesta rehabilitadora del Centro Histórico habanero y ofrecer a esta comunidad minoritaria, a la cual le son ajenos los mensajes de un medio de comunicación como la radio, la posibilidad de acercarse a las propuestas culturales e históricas que propone la otrora Villa de San Cristóbal de La Habana.

Asimismo, intenta potenciar un diálogo de retroalimentación con su cultura, la Sorda, con la pretensión de unificar fuerzas a favor de la preservación del patrimonio tangible e intangible de la nación; a la vez que promueve el conocimiento y uso de la Lengua de Señas Cubana (LSC), patrimonio de sus hablantes, para contribuir a su desarrollo en el ámbito cultural.

La falta de espacios socioculturales en Lengua de Señas Cubana para el desarrollo de esta con fines específicos, y la existencia del proyecto de desarrollo sociocultural en el Centro Histórico fueron otros de los motivos que animaron este ejercicio de comunicación.

Las acciones del programa van encaminadas a promover espacios de intercambio, de manera sistemática, sobre temas relacionados con la labor de los especialistas que de una forma $u$ otra inciden en el rescate de los teso- 
ros de la Nación, así como otros solicitados por los usuarios de la Lengua de Señas Cubana. Se contemplan además, la realización de recorridos guiados por el Centro Histórico habanero, acompañados por especialistas de la Oficina del Historiador que profundicen el acervo cultural de los Sordos a través del puente de comunicación que establece el servicio de interpretación. Por otra parte se adaptan materiales audiovisuales de carácter histórico y cultural producidos por Audiovisuales de la Oficina del Historiador, con la aplicación de la usc que guardarán la memoria histórica de su desarrollo y evolución, así como la preparación de otros que se expondrán en las salas permanentes de los museos del Centro Histórico, con el objetivo de hacer más accesible a ellos nuestros valores patrimoniales. Muy en breve se asumirá la aplicación del sistema de Closed Caption a programas televisivos como Andar La Habana, Entre libros, entre otros.

La impartición de clases de Lengua de Señas para los interesados en hacerse de las herramientas fundamentales de esta lengua, así como a aquellas personas cuya función es trasmitir información a los interesados: museólogos, especialistas, guías, etc. forma parte de este modelo de interacción entre las dos culturas.

3.1 VALORACIÓN DEL SISTEMA DE ACCIONES DEL PROYECTO CULTURA ENTRE LAS MANOS

El sistema de acciones que desarrolla el proyecto sociocultural Cultura entre las manos, intenta favorecer el acercamiento de nuevas colectividades al patrimonio habanero y nacional, a la vez que se propone responder a las principales demandas de los miembros de la comunidad lingüísticamente minoritaria de las personas Sordas, incidiendo en la elevación del nivel cultural de sus miembros, fomentando el desarrollo de la Lengua de Señas Cubana (LSC) y la inclusión social; ofreciéndoles plataformas posibles de actuación a fin de no reproducir las desigualdades sociales estructuradas que han definido su lengua y su cultura a través de los esquemas clasificatorios de la lengua dominante.

Por tanto el proceder que caracteriza cada una de las acciones de Cultura entre las manos, es muy consciente de que - de manera general - la solución de los problemas de un grupo vulnerable como el de las personas Sordas, es diferente cuando el análisis se da desde dentro del propio grupo de afectados, que cuando lo emiten personas sensibilizadas pero ajenas - por así decirlo - a la cotidianeidad de la comunidad en cuestión. Como ellos mismos confirman, las consideraciones de los protagonistas son máximas para el desarrollo del proyecto. 
"Ha habido un gran esfuerzo por todas las personas que participan en esto porque buscan lo que a nosotros nos interesa, las propuestas que hacemos, lo que queremos conocer. Nosotros quedamos muy complacidos siempre con lo que se nos ofrece $y$ es fundamentalmente, porque participamos activamente; se nos pregunta si nos gustó o no, para encontrar entre todos otra forma de llegar a nosotros si es que la que se usó no fue efectiva"-aseguró una persona Sorda de 61 años de edad, que cursó estudios hasta 12mo grado, habilitado como Mecánico montador y Pailero. Líder natural de la comunidad Sorda que durante varios años ha sido Presidente de la Asociación de Sordos de su Municipio de residencia, 10 de Octubre.

La relación intercultural estimulada por Cultura entre las manos garantiza el indudable enriquecimiento personal y colectivo, lo cual es posible porque, como afirma el MsC. Yoel Moya Pérez de Corcho, Sordopedagogo y Subdirector de Investigación y Desarrollo del Centro de Superación y Desarrollo del Sordo (CENSORD); "las acciones son concebidas, por primera vez, sobre la base del respeto a la diversidad comunicativa, cognitiva y cultural de la Comunidad Sorda Cubana y, especificamente la habanera". Ya que en la puesta en práctica de las mismas "se garantizan todas las condiciones necesarias para que las personas que participan sientan la satisfacción de aprender $y$ de disfrutar de espacios accesibles, además de propiciarles momentos de intercambio, de encuentro $y$ de socialización de sus experiencias personales”, asegura Moya.

La línea de trabajo que propone Cultura entre las manos se inscribe en el respeto a la pluralidad cultural, fundamentado en el reconocimiento de la comunidad Sorda y su lengua. "Los organizadores del proyecto saben que las personas Sordas tenemos necesidad de encontrarnos, compartir en nuestra lengua $y$ acceder a la información. Esta actividad logra cumplir todos estos anhelos porque nos toman en cuenta, escuchan nuestros criterios", comentó la MsC. Miriam Meneses, persona Sorda de 50 años, Jefa del Departamento de Comunicación y el Grupo de Investigación Nacional de la Lengua de Señas Cubana (LSC).

De la misma forma Meneses, reconoce la participación de los Sordos como elemento de peso para pasar de ser productos de las circunstancias a ser actores, contribuyendo a la eliminación de las barreras de comunicación: "el proyecto en sí, los cursos de Lengua de Señas que se están impartiendo al personal que trabaja en los Museos del Centro Histórico, esos videos que estamos filmando $y$ las otras actividades que se desarrollan, contribuyen a romper los muros comunicacionales con los que la comunidad Sorda se ha enfrentado históricamente, y lo que más nos satisface es que se hace desde nuestra visión.".

La Dra. Luisa América Toledo Martínez, Especialista en Sordos, Profesora Auxiliar de la Facultad de Educación Infantil de la Universidad de Ciencias 
Pedagógicas Enrique José Varona, destaca también otras aristas de la comunicación como el diálogo y el intercambio para el reconocimiento. "Las personas Sordas participan realmente y no son solo entes pasivos. Participan en la planificación de estas actividades porque tienen la posibilidad de proponer lo que les interesa, sus necesidades. Plantean sus inquietudes y el proyecto les da respuesta con acciones que realmente promueven en ellos el diálogo y el intercambio en su lengua. Acciones - continuó Toledo - dirigidas a conocer el entorno y la cultura que despiertan el compromiso con las tareas que desarrolla el propio proceso de la zona.".

Satisfacer los intereses y aspiraciones de los Sordos exige la accesibilidad a la información, lo cual es norte del trabajo de Cultura entre las manos. Como asegura Meneses, "las acciones concebidas son una forma de vincular a los miembros de la comunidad con la cultura en general y muy especificamente en lo que concierne al patrimonio, lo cual es de mucho interés para las personas Sordas. Así podemos todos acceder a esa información en nuestra forma de comunicación que es la Lengua de Señas Cubana", el elemento de comunión, su forma de comunicarse y conocer el mundo.

Cultura entre las manos es un espacio de cita, de reunión, de intimación como afirman miembros de la comunidad: "Yo asisto al proyecto por dos cosas, para ver a los Sordos y para aprender más. No vengo para que me anoten en una lista, ni por emulación, lo hago porque me interesa a mí", comentó una persona Sorda de 61 años, miembro del grupo de Comunicación de la Ansoc y Profesora de Lengua de Señas Cubana, graduada de $12 \mathrm{mo}$ grado y vecina del municipio Plaza de la Revolución.

"Primero nos vemos en la Plaza de las palomas, muchos llegamos temprano $y$ tenemos tiempo para saludarnos y conversar sobre algunas cosas, aunque también planeamos qué haremos más tarde."- agregó otro entrevistado de la comunidad de sorda de 48 años, residente en el Municipio Playa. Graduado como Técnico Medio en Edificaciones, labora actualmente como Rotulador en el Instituto de Ciencias Pedagógicas Enrique José Varona de Ciudad Libertad. "Después del proyecto algunos van a hacer las cosas de la casa, otros se quedan paseando por La Habana Vieja, se toman un café o almuerzan una pizza cerca, y si nos descuidamos nos agarra la noche en el Centro Histórico. Otros se van por ejemplo para Coopelia. Algunos vienen solos y encuentran una parejita como cosa de la juventud para hablar de amor y de cualquier otra cosa. Es un día que generalmente se aprovecha al máximo".

El encuentro y contacto de los Sordos cara a cara para expresarse, dialogar e interactuar en su lengua, resulta tan imperioso como cualquier otra necesidad legítima de una persona, los cohesiona como grupo. Como comentan un entrevistado, "...el Sordo busca al Sordo para poder comunicarse. Necesita 
estar donde está el otro Sordo para conversar en Lengua de Señas de infinidad de cosas, sobre amistades, trabajo, para hablar de deportes...."

La desarticulación de espacios de socialización habituales de la comunidad Sorda habanera ha provocado un improductivo distanciamiento entre muchos de ellos. "La Asociación de Sordos, que hasta hace algunos años estaba ubicada en Teniente Rey y Monserrate, Habana Vieja - hoy radicada en el municipio Playa -, ha provocado que disminuy a la concentración de personas Sordas en la sede, donde se reunían muchos Sordos por estar ubicada en un lugar céntrico. Desde entonces estamos muy dispersos y esto no es bueno para nosotros" - comentó una persona Sorda de 60 años, con estudios terminados de 12mo grado, y actualmente Instructor de Lengua de Señas Cubana del Municipio Plaza de la Revolución.

La experiencia de Cultura entre las manos es pionera en el trabajo con los Sordos en el Centro Histórico habanero. El modo en que los miembros de esta comunidad conocen y se apropian del mundo fue descubierto por hacedores de la restauración y otros especialistas, quienes - en su mayoría - ni siquiera habían tenido la oportunidad de pensar en la comunidad Sorda por primera vez. "Desprevenidos de todo tipo de preparación o prejuicio, esta iniciativa les ha permitido involucrarse en acciones desarrolladas desde la propia visión de sus integrantes: qué es para ellos Patrimonio, qué conceptos trabajar, cómo mostrarles lo realizado hasta ahora, cómo trazar nuevas metas de conjunto, cómo trabajar estos conceptos para los que a veces la lengua de señas aún resulta incompleta" - comentó la MsC. Yanet Rodríguez Martínez, integrante del grupo gestor del Proyecto como Especialista de la Dirección de Cooperación Internacional de la Oficina del Historiador.

La construcción de nuevas conexiones sociales por las que apuesta Cultura entre las manos para desarrollar en la persona Sorda toda una serie de conocimientos, hábitos y valores "estimula no solo las relaciones entre los Sordos, sino también de ellos con otras personas de la sociedad y con nuevas instituciones salidas del estrecho marco Escuela-ANSOC al que han estado acostumbrados", aseguró un especialista de la Asociación Nacional de Sordos de Cuba (ANSOc).

La comunicación posee carácter sistémico y no es solo una necesidad fortuita o contingente. La equiparación de oportunidades en este sentido es defendida por la convección de la onu por los derechos de las Personas Con Discapacidad ( $\mathrm{PCD})$, de la cual Cuba es firmante.

Este sector minoritario de la sociedad clama por la necesidad de unir fuerzas, de alcanzar la avenencia de criterios en su reconocimiento social para garantizar el control de la Comunidad Sorda sobre su cultura. Como ellos mismos expresan "...ojalá otros organismos se sumen a proyectos con estas características. Que nos tomen en cuenta, respeten nuestros criterios y respondan a nues- 
tras demandas, para que nos sintamos cada día mejor dentro de la sociedad", resaltó Meneses.

"Cultura entre las manos es compatible con los anhelos y los esfuerzos que hacemos nosotros para el desarrollo de la Comunidad Sorda - continuó - otro proyecto como éste no conocemos. Sabemos de algunas acciones que se han realizado en diferentes territorios de La Habana pero no se ha manejado como proyectos con un sistema de acciones coherente y consensuado con nosotros los Sordos".

Sobre la coherencia del trabajo del proyecto con la política educacional seguida por Cuba, expresó un Sordo entrevistado: "Estas acciones también contribuyen al desarrollo del modelo educativo bilingüe que se desarrolla hoy en las escuelas de Sordos por ser política educacional del país. Cultura entre las manos posibilita la extrapolación del modelo, o sea que el trabajo que alcanzaba solamente el contexto escolar y familiar, se puede decir ahora que también llega al plano social,y específicamente en relación con la cultura".

La construcción de conocimiento mediante la socialización es guía del trabajo de Cultura entre las manos. Esto se representa en primera instancia por la relación de las personas Sordas con otros actores del proyecto, especialistas en las diferentes materias como: Patrimonio, Comunicación, Gestión Cultural, Historia, Economía, Arquitectura, entre otros; así como con sus Intérpretes, aquellos que establecen el puente entre ambos grupos y el patrimonio. A esto se refiere la MsC. Onedys Calvo, Especialista en Artes Visuales de la Oficina del Historiador, quien describe el sistema de acciones del proyecto como "oportuno y abierto por haber logrado implementar varios niveles de comunicación”. Cita el caso de los encuentros mensuales como un espacio en el que los participantes "descubren temáticas específicas y actores determinados, donde se trabaja de manera intima y cómoda porque se ofrece una comunicación inmediata con un individuo (especialista en una materia determinada) sobre cuestiones definidas"; menciona los recorridos "en los que guiados por un entendido en la materia que se aborda pueden moverse por diferentes instancias de la ciudad, y cuya selección ha sido muy acertada, tomando en cuenta las referencias culturales que poseen las personas Sordas, la capacidad de interactuar y de participar por el propio concepto de Rutas y Andares". Igualmente Calvo se refirió a los materiales audiovisuales elaborados por Cultura entre las manos empleados como "alternativa de comunicación más inmediata, ajustada a la medida de la Cuba de hoy porque en términos generales hoy la cultura es más audiovisual" y como última cuestión la especialista hace alusión a los cursos de Lengua de Señas como impulsores de la interacción social y la socialización de la Lengua de Señas, potenciándola como elemento gestor del desarrollo que entre otras cosas, puede influir en el bienestar de las actuales y futuras generaciones. “...han permitido que más personas oyentes 
se involucren en la adquisición de nuevos saberes como el aprendizaje de la Lengua de Señas, una lengua que pudiéramos aprender todos, por lo menos para comunicarnos dentro de la frontera". Y, concluyó diciendo, "pienso que más allá de mirar las acciones como una oportunidad que se abre, estas se sistematizan en un proyecto de integración”.

\subsection{EVOLUCIÓN DEL NIVEL DE INFORMACIÓN Y CONOCIMIENTO EN LA COMUNIDAD SORDA, SOBRE CUESTIONES DEL PATRIMONIO TANGIBLE E INTANGIBLE}

Cultura entre las manos es una experiencia de comunicación para el desarrollo que ha incidido considerablemente en la evolución del nivel de información y conocimiento de la comunidad Sorda sobre cuestiones del patrimonio tangible e intangible así como en otras temáticas más generales relacionadas con el acontecer nacional e internacional. Asimismo, ha aumentado la capacidad expresiva y de diálogo de la comunidad Sorda y ha creado nuevas formas de integración grupal y social entre ella y la gran mayoría de personas oyentes.

La impronta del proyecto Cultura entre las manos y sus acciones en la comunidad Sorda han determinado un incremento del nivel de conocimiento de sus actores que se ha traducido como ellos mismos reconocen en mayor enriquecimiento y posibilidades de acceso a la cultura. "En los inicio del proyecto, cuando empezamos a participar algunos no teníamos conocimiento de lo que era el patrimonio. Ahí fue cuando comenzamos a adentrarnos un poco más en el aprendizaje de la historia que es muy bonita porque cada pedacito de La Habana la tiene. Eso ha influido en el desarrollo cultural de nosotros, lo ha ampliado, nos ha hecho entender la cultura $y$ su importancia. Cuando ahora pasamos por un lugar, observamos un óleo o una escultura ya podemos comprender que todo tiene su historia $y$ transmite cultura. Eso lo hemos aprendido con el proyecto" - aseguró un miembro de la Comunidad Sorda participante habitual del Proyecto.

Asimismo, otros miembros de la comunidad Sorda que asisten regularmente a las acciones del Proyecto y se identifican con sus propósitos sintiéndolos como suyos, han confirmado que "este proyecto es muy importante para los Sordos porque nos ayuda a conocer la historia, la cultura cubana que al final es nuestra identidad, nos informa, nos actualiza. Los Sordos estamos muy cerrados a la información. Nuestros padres no nos informan, nuestros amigos tampoco por eso pienso que el proyecto es como si fuera la mamá y el papá de nosotros. Nos enseña muchas cosas, nos abre los ojos ante mucha belleza. Es un proyecto de nosotros".

Sobre la impronta del proyecto en el incremento de los niveles de conocimiento, los actores del Proyecto consideran que participando en Cultura entre 
las manos se aprende mucho. "Hay cosas que no sabía sobre la historia del automóvil por ejemplo, o el vestuario de época, y si conocía sobre ello no era con la profundidad con que se tratan los temas en el proyecto. Para mí todos son importantes, con todos aprendo. Yo puedo leer - continuó - pero aun así participando en el proyecto aprendo muchísimo porque lo recibo todo en mi lengua. La mayoría de los Sordos no leen por lo tanto la información en lengua de señas es trascendental".

De igual manera, eliminar las barreras de comunicación constituye una plataforma indispensable para el intercambio cultural. Una persona Sorda de 28 años, Lic. en Interpretación de la Lengua de Señas, Instructora de Lengua de Señas del Municipio Habana del Este, quien ha estado muy cerca de Cultura entre las manos desde sus inicios como voluntaria, agregó: "El proyecto ofrece una posibilidad de comunicación con la que antes no contábamos. Ha sido como una apertura", pues aunque la gran mayoría de la sociedad interactúa y encuentra la manera de satisfacer su necesidad de conocimiento a través de la escuela, los medios de comunicación, en el hogar y las prácticas sociales, la comunidad Sorda presenta otra realidad que se traduce en maneras distintas de acceso a la información.

“...Es un sector de la sociedad que, generalmente, no recibe una atención cultural óptima para llegar plenamente a la información y a los conocimientos. La Comunidad Sorda cubana históricamente recibe su formación y desarrollo cultural en las instituciones educativas especializadas pero fuera de estos contextos se ve muy limitada para continuar ampliando y enriqueciendo su acervo, lo cual hace que sus integrantes conciban y desarrollen sus propios espacios y que los elementos culturales e identitarios sean cada vez más marcados y restringidos. Este tipo de proyecto facilita una ampliación y diversificación en los horizontes culturales de las personas Sordas", como aseguraron especialistas de la ANsoc.

Si se habla de información especializada las posibilidades de acceso son aún más difíciles, ya que esta se encuentra habitualmente fragmentada o dispersa. Facilitar el acceso a ella interactuando con el entorno y propiciar el dialogo sobre las temáticas más disímiles, es la tarea de los especialistas invitados o los guías convenidos para participar en las acciones del Proyecto.

De aquí que Onedys Calvo, historiadora del arte vinculada al proyecto, explicó que "...esta es una oportunidad otra para hacerse de la información. En primer lugar porque existe la motivación para salir a un lugar determinado, a disfrutar de una experiencia que además le va a aportar otro tipo de conocimiento. Es un espacio al que no sólo acuden las personas Sordas a aprender algo sino a socializar, a disfrutar buscando en grupo una comunidad de intereses en otro tipo de conocimiento. $O$ sea, que desde este punto de vista es otro tipo de información $y$ otra manera también de recibirla, de una forma mucho más amena, participativa e 
interactiva. El proyecto ha sido como una apertura a diferentes dimensiones y que cada uno asumirá según sus propias experiencias individuales pero estoy segura que es un espacio revelador que les enseña, que descubre, que incita".

Afirmación coincidente con la expresada por, Magda Resik, directora de Habana Radio, quien piensa que "en este proyecto se está logrando que esa comunidad a la vez que participa aprenda mucho más sobre su entorno, se reconozca mucho más en los bienes patrimoniales, en los procesos culturales, $y$ en determinado fenómeno social. El hecho es que ya esa comunidad está participando, generando procesos de aprendizaje colectivo porque el proyecto no sólo propone la enseñanza de la lengua de señas, también plantea que desde la comunidad se gesten sus propios contenidos a comunicar y a promover con la participación de esa comunidad".

El Proyecto posee la capacidad de generar procesos de aprendizaje colectivo en la comunidad Sorda, tal es así que en ocasiones, luego del tratamiento de un tema específico, líderes de la comunidad han organizado actividades para profundizar aún más en lo aprendido. Entre ellas se pueden citar la visita al Planetario y al Dpto. de Closed Caption del Instituto Cubano de Radio y Televisión (ICRT).

Atendiendo a los criterios de la concepción antropológica de la cultura que sistematiza Yúdice (2009) en su estudio, este ejercicio de comunicación se propone incorporar conocimiento, valores, normas y costumbres en la comunidad habanera que participa, como vía para llegar al desarrollo. "Cada nuevo encuentro, cada acción contiene un gran volumen de información, que se ofrece de manera tal que pueda estar al alcance de la heterogeneidad del grupo que asiste, - afirmó la MsC. Carmen Salgado Castillo, Sordopedagoga y Asesora e Intérprete del Proyecto -, considero que es un proyecto de pueblo para generar desarrollo intelectual. Un proyecto que actualiza, muestra nuestras raíces, educa sobre quiénes somos y hacia dónde vamos los cubanos, incentivando el amor por nuestra Cuba, $y$ eso es desarrollo.”.

La transversalidad de la cultura y su interrelación con las diferentes áreas, enaltece la naturaleza cultural del patrimonio y su valor como propiedad y responsabilidad colectiva, especialmente en un espacio donde son revelados nuevos significados y prácticas mediante la relación intercultural que propicia la gestión de Cultura entre las manos.

\subsection{VALORACIÓN DE LA CAPACIDAD EXPRESIVA Y DE DIÁLOGO ADQUIRIDA}

Cultura entre las manos goza de la posibilidad de reunir un significativo número de Sordos adultos. Personas con necesidades comunicacionales similares, así como niveles de desarrollo cultural promedios, miembros de la Asociación 
de Sordos o no, que han concluido su paso por las instituciones educativas; únicos contextos de participación colectiva en los que interactuaban antes de surgir el proyecto. "Cuando empezó Cultura entre las manos, esto era una cosa muy nueva para nosotros - comentó una persona Sorda - . Ha pasado el tiempo, ha crecido el proyecto y hemos crecido nosotros con él. Siempre tenemos la posibilidad de decir lo que pensamos, hacer preguntas, de expresarnos, ser nosotros mismos, y eso nos hace sentir muy bien. Además, nadie de los que viene se va con dudas. Usted levanta su manito y pregunta de cualquier aspecto y se le explica con tremenda calidad $y$ amor. Esto ha influido por supuesto en que la gente está más preparada, más informada, $y$ más segura de sí".

La ampliación del escenario de actuación que propicia esta práctica comunicativa, constituye una nueva opción cultural para la comunidad Sorda. Un espacio diferente donde se ayuda a satisfacer la necesidad de fortalecer capacidades de participación en otro contexto.

La oportunidad de dialogar con especialistas en las más disímiles materias y acercarse a lo que en algún momento les parecía inalcanzable como intercambiar cara a cara con personalidades de la cultura como el Dr. Eusebio Leal Spengler, la Dra. María Dolores Ortiz, el Historiador y periodista Ciro Bianchi; igualmente conversar con actrices como Yanay Penalba, quien interpretó el papel de la muchacha Sorda en el Tele play "Esta vez es la vez", audiovisual que trata el fenómeno de la integración de la persona Sorda, producido por la División de Programas para la Niñez y la Juventud de la Televisión Cubana; disfrutar de la película cubana Habanastation con el servicio de interpretación en Lengua de Señas, así como de la presencia de los protagonistas del filme para compartir impresiones; visitar las Casas Museo del Centro Histórico y recibir información de primera mano sobre las muestras permanentes y transitorias, la historia de las edificaciones, etc.; cuentan entre las nuevas experiencias culturales sobre las cuales dialogar, que han enriquecido el conocimiento, y contribuido al fortalecimiento de la capacidad expresiva y la participación.

Como apuntan los miembros de la Comunidad Sorda que participan en el proyecto "a través de las informaciones que hemos recibido en las acciones del proyecto, en la voz de los propios especialistas y personalidades, hemos podido aprender cosas nuevas. Indudablemente esto nos ha hecho expresarnos mucho mejor sobre muy variados temas ante amigos y cualquier otra persona con la que hablemos. Ya podemos explicar más sobre la historia de Cuba - nuestro país -, de La Habana y sus lugares históricos importantes como por ejemplo, El Templete, que es el sitio fundacional y la historia de los nombres de algunas de las calles de la ciudad con conocimiento, algo que anteriormente no podíamos hacer porque no teníamos la información, estábamos truncados, bloqueados". 
Las posibilidades de diálogo, estimuladas por Cultura entre las manos son referidas por muchos de los participantes. Los miembros de la comunidad confirman el impacto del proyecto en su relación familiar: "Mi hija por ejemplo, no tiene mucho tiempo y tampoco le gusta levantarse temprano el sábado porque se pasa la semana en la Universidad, pero siempre que regreso del proyecto me pregunta sobre qué se trató el encuentro, el recorrido, o lo que se haya hecho. La última vez - continuó el entrevistado - le tuve que contar todo lo que aprendimos en la visita al Castillo de la Fuerza, y le hablé entonces sobre los 3000 barcos hundidos que tenemos en el mar de Cuba desde hace siglos y que no se han podido extraer, ya que no contamos con los aparatos modernos para eso".

Los participantes del proyecto tienen la posibilidad de convertirse lo mismo en promotores del proyecto que en trasmisores del conocimiento, lo cual en muchos casos genera una nueva retroalimentación. Aspecto sobre el que también expresan: "hay familiares y otros amigos con los que conversamos que tienen algún conocimiento sobre la época o el tema del que se conversó en el proyecto, $y$ con estas personas se produce un nuevo intercambio donde nos sentimos muy cómodos porque podemos aportar".

La Comunidad Sorda destaca que lo aprendido ha servido también para motivar a la nueva generación. "Lo que aprendemos en el proyecto es lo que le transmitimos nosotros a los jóvenes para que conozcan nuestra historia $y$ además se motiven a visitar el Centro Histórico. Les hablamos de las Casas Museos y lo que pueden encontrar en ellas. Les hacemos como una invitación con lo que sabemos porque ya estamos preparados para esas cositas.".

La concatenación de acciones del proyecto ha contribuido a la asociación de contenidos. Como plantea la MsC. Marianela Garau Cordobés, Lingüista y Coordinadora de la Licenciatura en Interpretación de la Lengua de Señas, de la Universidad de La Habana "por la manera en que participan en el debate haciendo preguntas muy atinadas sobre un tema especifico para lograr más información y conocimiento, podemos medir la manera cómo integran el contenido recibido en las diferentes acciones".

La Lengua de Señas es una lengua conversacional, una lengua de uso totalmente ágrafa, que en Cuba aún necesita enriquecimiento y desarrollo. La Lengua de Señas Cubana como fenómeno vivo de la comunicación, progresará en la medida en que se emplee en los más variados contextos. Cultura entre las manos es un espacio creado precisamente para la socialización de esta lengua en un escenario prácticamente virgen para ella, la cultura y el patrimonio, un nuevo ámbito para colectivizarla y compartir sus avances. "En el proyecto hasta se aprenden nuevas señas - explicó una persona Sorda - lo mismo porque las personas mayores dominan una Lengua de Señas anti- 
gua que ya no se usa y tienen entonces la oportunidad de aprender las modernas, las oficializadas, $y$ ver los cambios que han sucedido, que por una negociación lingüística que es necesaria hacer momentáneamente porque la seña no existe o el concepto no se conoce, asi es que de todas formas enriquece nuestro vocabulario aportando nuevos conceptos. A mí, que no soy tan viejo me pasa también, y es porque la Lengua de Señas es una lengua viva, que crece e incorpora otras señas necesariamente".

La incursión de Cultura entre las manos en temáticas tan específicas ha puesto en evidencia la necesidad de desarrollo de la Lengua de Señas Cubana, partiendo del hecho de que un concepto que no esté en la lengua de una cultura es la resultante de que esa cultura no ha necesitado ese concepto y que podría incorporarlo si esta surgiera.

A propósito algunos de los expertos y especialistas entrevistados afirman. "El proyecto ha obligado a ampliar el léxico de la Lengua de Señas Cubana para dar respuesta a la falta de señas para el abordaje y comprensión del contenido o la información que se recibe en el ámbito de la cultura y el patrimonio. Ha impuesto una necesidad y como consecuencia el patrimonio lingüistico ha crecido".

La integración de las acciones del proyecto como sistema ha venido a enriquecer su funcionamiento para el logro de los objetivos. Como asegura Yanet Rodríguez, miembro de grupo gestor del Proyecto,"para contribuir al desarrollo lingüistico de esta comunidad se necesitan varias acciones integradas, no elementos aislados, $y$ esto es lo que ha generado el Proyecto. Ha sido una punta de lanza en el desarrollo lingüístico de esta colectividad a partir de la organización del trabajo".

El proyecto ha logrado estimular procesos participativos, no solo por el hecho de que la persona sorda siente que está, sino porque es capaz de generar procesos de aprendizaje colectivo. Y esto viene dado por el "carácter natural, voluntario y estimulante" con el que se ha trabajado durante estos cinco años, para contribuir a "que las personas Sordas encuentren un espacio para expresarse en diferentes momentos $y$ de muy variadas formas, para formar parte de algo que ya han hecho suyo", lo cual se demuestra sin dudas, en la elaboración de los mensajes de bien público que de conjunto con estudiantes de Comunicación Visual, del Instituto Superior de Diseño (ISDI), más tarde fueron llevados al discurso gráfico.

\subsection{NIVELES DE INTEGRACIÓN GRUPAL E INCLUSIÓN SOCIAL ALCANZADOS}

Cultura entre las manos se inserta en la línea de trabajo de la Oficina del Historiador que enmarca un proceso de rehabilitación de un Centro Histórico patrimonial en un fenómeno social muy singular, que pretende su población 
sea la mayor beneficiaria del proceso de restauración. Así, la comunidad Sorda, igualmente encontró en este sitio un lugar para progresar y crecer espiritualmente, teniendo la oportunidad de apropiarse - en su lengua - del legado cultural habanero y cubano, que también es suyo.

$\mathrm{Y}$, es que el patrimonio por el que trabaja cada día la Oficina del Historiador, - espejo de la identidad nacional - como asegura Orlando Inclán Castañeda, Arquitecto de la Dirección General de Proyectos de Arquitectura y Urbanismo de la Oficina del Historiador, "no puede vivir sin las personas de todos los grupos sociales. Si no se conoce no se respeta, no se cuida, no se le aporta, y si no se le aporta muere porque sería un objeto vacío, solo museable, sin valor, pues precisamente ese valor está dado por lo que las personas le confieren".

Llegado a este punto es importante referirse a las posibilidades que desde esta otra perspectiva propicia el proyecto. A esto se refieren otros actores como la MsC. Martha Oneida Pérez Cortés, Jefa del Grupo de Investigaciones del Plan Maestro de la Oficina del Historiador, cuando explica: "como parte de la comunidad oyente, puedo decir que en ocasiones no vemos más allá de lo que nos sucede a nosotros mismos. Pero, este proyecto ha puesto a Andar La Habana a otro grupo de personas en las cuales también hay que pensar. No quiere decir que no supiéramos que existen las personas Sordas, pero el trabajo que realiza el proyecto con ellos como comunidad, contribuye a que visionemos muchas otras cosas, a que nos digan: estamos aquí, está propuesta no es accesible para nosotros, qué se puede hacer".

No solamente depende de las personas Sordas el hecho de llegar a ser incluidos en la sociedad, también quienes conforman la mayoría deben estar listos para ello y trabajar para lograrla. Una de las formas posibles es precisamente preparándola, no solamente para que acepte, sino también para que se pueda acercar a esa persona y lograr un nivel de comunicación con ella. Aspecto en el que Cultura entre las manos ha intentado poner su granito de arena, al menos en el entorno del Centro Histórico habanero de manera directa."Cultura entre las manos - aseguran los expertos - nos ha permitido ver nuestro trabajo en otra dimensión, más allá de la limitación del lenguaje. Está logrando que la comunidad Sorda se integre alrededor de procesos creativos, comunicativos, pedagógicos y educativos; incluso está proponiendo una integración que va más allá de un simple encuentro social, es una integración que en sí misma genera comunicación, desarrollo y participación".

El vínculo o la interacción entre ambos grupos - la gran mayoría y la comunidad Sorda -, pueden garantizar la formación de criterios de accesibilidad en función de las necesidades de este grupo, y así lograr ofertas más apegadas a sus demandas. 
Lacomunidad Sorda puede aportar mucho a la sociedad. Y si participa esencialmente buscando el conocimiento, explorando más sobre de dónde venimos, cómo somos, cómo nos expresamos, reconociendo la historia patria, y todos esos valores de los que somos portadores los cubanos se está poniendo en práctica una buena manera de incluirla en la sociedad toda.

$\mathrm{El}$ proyecto reconoce y pone al descubierto potencialidades de las personas Sordas, estimula que las propias personas de esta comunidad evidencien que las tienen y que las pueden poner al servicio de la sociedad toda, lo mismo en condiciones laborales, en ocasiones de creación, que en la elaboración de mensajes comunicativos.

Cultura entre las manos se ha convertido en la herramienta que las personas Sordas tienen para acercarse al entorno cultural y patrimonial de la urbe habanera, lo cual es valorado por Yanet Rodríguez, especialista de la Oficina del Historiador de la Ciudad de La Habana, como satisfactorio para contribuir a su inclusión social:"iniciativas como Cultura entre las manos, estimulan la sensibilidad de la población en general y ayudan a la integración social de un grupo de la población, que aunque minoritario forma parte también de un territorio, que aspira a un desarrollo local determinado y para ello es necesaria la inclusión de todos en los procesos de construcción social".

Pero, esta relación cultural no se consolida sólo desde la sensibilidad de la mayoría ante la diferencia, se asegura realmente mediante el compromiso social. Con modos de actuación que partan de posiciones de igualdad, propiciando procesos comunicativos y participativos que satisfagan las disímiles situaciones que impone la diversidad.

Una especie de llamado a la conciencia puede ser el trabajo de Cultura entre las manos, expresado en el cambio de perspectiva de algunos de los organizadores de probados Programas de la Oficina del Historiador (он). Las Consultas Públicas y Rutas y Andarespara descubrir en familia, dirigidos por el Plan Maestro y la Dirección de Programación Cultural de la oH respectivamente, fueron de los primeros en dar muestras de ello.

Con el propósito de recoger sus opiniones la Consulta Pública sobre el Plan Especial de Desarrollo Integral (PEDI) del Centro Histórico, llegó por primera vez a la comunidad Sorda en 2011. A solicitud de sus coordinadores Cultura entre las manos preparó un Encuentro especial para incluir la opinión de este grupo social, tomando como muestra a los actores del proyecto, quienes en su mayoría no viven en el Centro Histórico, pero pueden garantizar una más amplia visión a los encargados deactuar en pos de la accesibilidad y el desarrollo integral en la urbe habanera.

$\mathrm{Y}$, es que Cultura entre las manos ha funcionado - se puede decir - como 
un pequeño motor que impulsa a los encargados de la gestión patrimonial a pensar en las posibilidades reales que poseen sus proyectos para hacerlos accesibles a las personas Sordas, y en consecuencia actuar.

\section{CONCLUSIONES}

El desarrollo de la comunidad Sorda habanera ha sido intervenido desde el punto de vista cognitivo luego de implementarse las prácticas de comunicación potenciadas por Cultura entre las manos. Un proyecto intercultural que como ejercicio facilitador de la mediación social, ha incidido tanto en la integración de la comunidad Sorda habanera tal y como se describió en publicaciones anteriores, como en la producción de una comunicación personalizada basada en la interacción cara a cara, y el respeto a los códigos culturales del público al que está dirigida. Así, incentiva la elevación del nivel cultural en su sentido más amplio, y la motivación por el conocimiento sobre temáticas antes no exploradas, o revisitadas desde perspectivas diferentes.

De aquí que el proyectono discrimina sino promuevela diferencia, transforma la diversidad en una ventaja, un valor, una oportunidad y un derecho. Toma en cuenta las demandas, necesidades, posibilidades y recursos propios de una comunidad antes no incluida en la vida cultural del Centro Histórico habanero.

De esta forma, la comunidad Sorda como grupo culturalmente diferente, mediante el intercambio entre ellos y con la mayoría oyente pone en escena su "yo", generando incluso otras formas de percepción de uno mismo. Ha logrado significar la realidad de forma participativa. En este caso enriquecida a través de la retroalimentación producida por ese toma y da de grupos culturalmente diferentes, que permite la construcción y reconstrucción de ambas realidades.

La experiencia de cada uno de los miembros de la comunidad Sorda como la de todo ser social, se enriquece de forma diferente. El punto de partida siempre es la individualidad, y por lo tanto la diversidad de realidades e historias que poseen.

La integración o inclusión social del Sordo, es otro capítulo más extenso en sus vidas, por el que se está trabajando hoy con Cultura entre las manos. De aquí que las necesidades, intereses, motivaciones, aspiraciones y expectativas de cada una de las personas Sordas son diferentes. Por tanto, el conocimiento y las nuevas conexiones sociales e Institucionales que ha propiciado el proyecto, son entendidos y aprovechados por cada uno de los actores de múltiples maneras. 
A partir de la configuración y el fortalecimiento de las nuevas redes como soporte para conquistar un espacio otro en la vida social y la confianza, el cambio ha sido no solo individual sino también colectivo. Algunos han comprendido el contexto y han reconocido su uso apropiándose del conocimiento, del espacio como ámbito para recrear la identidad, de los nuevos vínculos, etc.

La identificación de un patrón de relación donde las personas y las Instituciones interactúan ha hecho que conciban el Centro Histórico habanero como algo suyo sumándose a concursos promovidos por las Casas Museo del lugar de manera independiente, otros apuestan por ganar premios participando en las actividades del mes con sus amigos y colegas; y otros muchos prefieren esperar a que el proyecto realice sus actividades para así satisfacer sus intereses. También está el caso de quienes matriculan en talleres como el de la Boligrafía; este último aunque no es promovido, ni desarrollado por la Oficina del Historiador, se cita entre los ejemplos por haber sido motivo de atención de varios miembros de la comunidad Sorda después de su participación en Cultura entre las manos.

Igualmente, en esta nueva relación con el contexto del Centro Histórico habanero, es posible apreciar que una segunda comunidad de intereses los une alrededor de los tesoros patrimoniales. Un buen número de ellos muestra mayor deseo por aprender por sí mismos, actualizarse sobre lo que acontece nacional e internacionalmente, y sobre todo por conocer otros aspectos de la vida en sociedad, y de la historia de su país evidenciándose un incremento de las áreas de información y conocimiento, ya que se sienten útiles teniendo algo más que aportar tanto en el marco social como familiar.

Acudir al Centro Histórico - como contexto sociocultural de prestigio que hoy los incluye -, conlleva a que se arreglen y vistan para un día especial, al que no faltan a no ser por razones mayores. Es en definitiva, un espacio concebido por ellos - en primera instancia - como lugar de la cultura propicio para sus citas y encuentros; en segundo lugar espacio de disfrute en grupo de actividades especiales en su lengua, que entre otras cosas propicia el diálogo con voces autorizadas y personalidades de la cultura como iguales; $\mathrm{y}$ en tercer lugar un sitio desde donde planear otros paseos para aprovechar el día.

Así, la necesidad de las personas Sordas de interactuar cara a cara para dialogar en su lengua - en un espacio de alta significación cultural -, es satisfecha por esta alternativa de comunicación y lo que de ella se deriva.

El cara a cara no tiene una connotación tan elevada para los oyentes como para los Sordos. Las características de la Lengua de Señas, así como el nivel 
de desarrollo tecnológico del que puede disponer un país en camino al desarrollo, son algunos de los elementos que hacen de esta la mejor vía de comunicación para la comunidad Sorda habanera.

Cultura entre las manos ha ofrecido un espacio para la interacción que ha incidido en la satisfacción de intereses culturales de la comunidad Sorda, en su visibilización en un marco social donde antes no se ubicaba, lo cual ha garantizado que además sea reconocida por otros que ni siquiera pensaban en ella porque tampoco la conocían.

La construcción de conocimiento estimulada por Cultura entre las manos mediante la socialización se ha traducido en mayor presencia en la vida pública del Centro Histórico, participación y posibilidades de acceso a la cultura. Pero, este beneficio no ha sido en una sola dirección, la retroalimentación cultural ha ocurrido en dos sentidos. Los especialistas - actores del proyecto - también han ampliado su panorama sobre la realidad social y han conocido otras posibilidades de proyección en temas de accesibilidad.

\section{BIBLIOGRAFÍA REFERENCIADA}

BELTRÁN, L.R. (2005). La comunicación para el desarrollo en Latinoamérica: un recuento de medio siglo. Paper presented at the III Congreso Panamericano de la Comunicación. Buenos Aires. Argentina.

CASTELLANOS, R.M.; RODRÍGUEZ, X. (2003). Actualidad en la educación del niño sordo. La Habana: Pueblo y Educación.

DOMÍNGUEZ, D.M. (1994). Un breve análisis de la Lengua de Señas Cubana. La Habana: Universidad de La Habana.

GALINDO, J.C. (1998). Técnicas de Investigación en sociedad, cultura y comunicación. D.F: Addison Wesley Longman.

MASSONE, M.I.; MARTÍNEZ, R.A. (2012). Curso de Lengua de Señas Argentina. Parte I. from http://www.cultura-sorda.eu/resources/Massone Martinez_Curso_LSA_PARTE_I_2012.pdf.

OROZCO, G. (2002). Mediaciones tecnológicas y des-ordenamientos comunicacionales. Signo y Pensamiento.

PÉREZ, H. (2004). Estudio lingüístico de los parámetros formativos quinésicos de la lengua de señas de los escolares sordos, en el área de Química. La Habana: Instituto Superior Pedagógico Enrique José Varona.

SERRANO, M.M. (2008). La mediación social (Edición conmemorativa del 30 aniversario (1 $1^{\mathrm{a}}$ Edición. 1977; $2^{\mathrm{a}}$. 1978. Reediciones: 1981-1983-1986). Madrid: Akal.

VALENCIAGA, C. (2003). Programa de Lengua de Señas Cubana para maes- 
tros, intérpretes y padres de niños sordos. La Habana: Instituto Superior Pedagógico Enrique José Varona.

VALENCIAGA, C. (2005). Caracterización del parámetro lingüístico-quinésico: configuración manual de la lengua de señas cubana (LSC). Paper presented at the VIII Congreso Latinoamericano de Educación Bilingüe para sordos, La Habana.

YÚDICE, G. (2009). Los conceptos: Desarrollo, Sostenibilidad y Cultura Paper presented at the Cultura y Desarrollo: América Latina frente al desafío de un desarrollo culturalmente sustentable. Conferencia dictada en el marco del Postgrado on-line: Gestión del Patrimonio y Comunicación. Argentina: FLACSO. 